\title{
Rogue waves and rational solutions of the nonlinear Schrödinger equation
}

\author{
Nail Akhmediev, ${ }^{1}$ Adrian Ankiewicz, ${ }^{1}$ and J. M. Soto-Crespo ${ }^{2}$ \\ ${ }^{1}$ Optical Sciences Group, Research School of Physics and Engineering, Institute of Advanced Studies, The Australian National University, \\ Canberra, Australian Capital Territory 0200, Australia \\ ${ }^{2}$ Instituto de Óptica, CSIC, Serrano 121, 28006 Madrid, Spain
}

(Received 19 May 2009; published 4 August 2009)

\begin{abstract}
We present a method for finding the hierarchy of rational solutions of the self-focusing nonlinear Schrödinger equation and present explicit forms for these solutions from first to fourth order. We also explain their relation to the highest amplitude part of a field that starts with a plane wave perturbed by random small amplitude radiation waves. Our work can elucidate the appearance of rogue waves in the deep ocean and can be applied to the observation of rogue light pulse waves in optical fibers.
\end{abstract}

DOI: 10.1103/PhysRevE.80.026601

PACS number(s): 42.65.-k, 47.20.Ky, 05.45.Yv

\section{INTRODUCTION}

Rational solutions of the nonlinear Schrödinger equation (NLSE) play a major role in the theory of rogue waves [1,2]. There is a hierarchy of them with progressively increasing central amplitude. The first-order rational solution was given by Peregrine [3] as early as 1983 . The next-order one, based on [7], was recently presented in [1] as a possible explanation for rogue waves with higher amplitude. Simple interactive demonstrations are readily available [4]. In the present work, we give a technique for the systematic derivation of the whole hierarchy of these rational solutions. As examples, we consider the four lowest-order solutions and show that even the third-order one can appear in numerical simulations with random initial conditions.

The major reason supporting the claim that rational solutions are important in the composition of ocean waves is that, as with waves created by modulation instability, they "appear from nowhere." In other words, the instability induced from a small perturbation on top of a plane wave leads to an increase in the perturbation up to its highest amplitude and then to a decay so that it finally "disappears without a trace." Although for rational solutions the growth rate of the instability is zero, they still develop according to a power law. This may appear to take a very long time, but on the other hand, the ocean is vast and there is enough space for the development of even such slow instabilities.

Rational solutions are limiting cases [5] of either periodic "Ma solitons" (MS) [6] or "Akhmediev breathers" (ABs) [7-11]. MSs have to be created directly from initial conditions consisting of the background plane wave+soliton. In other words, Ma solitons must exist in the wave field right from the very beginning. Only rational solutions and ABs belong to the class of excitations that appear from nowhere $[1,10]$. ABs, in particular, arise during evolution due to modulation instability $[7,12,13]$. Once they have appeared, they may collide just as can happen with solitons. Collisions of two or more ABs with transversal frequencies close to zero may create structures similar to higher-order rational solutions [11]. Thus, studies of rational solutions of higher order are of fundamental importance. They may resolve the mystery of rogue waves in the ocean [14-16] and help in creating useful rogue waves in optical fibers $[17,18]$.
As in two of our previous papers [1,11], we deal here with the standard "self-focusing" NLSE. In the dimensionless form, it is given by

$$
i \frac{\partial \psi}{\partial x}+\frac{1}{2} \frac{\partial^{2} \psi}{\partial t^{2}}+|\psi|^{2} \psi=0,
$$

where $x$ is the propagation variable and $t$ is the transverse variable. The notation here for independent variables $x$ and $t$ is reversed in comparison to the convention taken in the classical work by Zakharov and Shabat [19]. However, our convention is standard in the theory of ocean waves [20] and waves in optical fibers [21] so we use it throughout this paper. In each case, the function $\psi$ describes the envelope of the modulated waves, and its absolute value carries information about either wave elevation above the water surface or the intensity of optical wave.

As stated, we are interested in solutions of NLSE (1) that can be expressed in the form of a ratio of two polynomials. Thus, they are called "rational solutions." This form of solution always appears with a multiplicative exponential factor, $\exp (i x)$, so that the full solution may not be rigorously termed "rational." However, this factor disappears when the absolute value of the function $\psi$ is calculated and so is irrelevant from a physical point of view.

Some solutions involving polynomials have been given for the "self-defocusing" NLSE [22,23]. We stress that the latter solutions are for a different equation. Besides, they all contain singularities and thus do not represent physical situations. In contrast, all solutions that we present here are finite everywhere and thus can and do represent real physical waves.

\section{TRADITIONAL DARBOUX TRANSFORMATION SCHEME}

The general scheme for solving the NLSE from given initial conditions is the inverse scattering technique [19]. Certain classes of solutions can also be constructed with the use of dressing methods. A particular case of the latter is the so-called "Darboux transformation" (DT) [24]. The DT can be used to construct multisoliton solutions or solutions involving several ABs [11]. Solutions of each class consist of a corresponding "hierarchy" of solutions. 
Rational solutions belong to a special class and generally cannot be constructed using the traditional DT technique. The lowest-order rational solution or "Peregrine" soliton can be obtained either as a limiting case of a Ma soliton or an AB. Obtaining higher-order rational solutions in a similar procedure would be highly involved although not completely impossible. Thus, we have modified the DT technique in such a way that limits are taken in the intermediate calculations. This modification has allowed us to find a way to construct the whole hierarchy of rational solutions.

The NLSE is a condition of compatibility of the two following linear equations:

$$
\begin{gathered}
\mathbf{R}_{t}=l \mathbf{J R}+\mathbf{U R}, \\
\mathbf{R}_{x}=l^{2} \mathbf{J R}+l \mathbf{U R}+\frac{1}{2} \mathbf{V R},
\end{gathered}
$$

where $\mathbf{U}, \mathbf{J}$ and $\mathbf{V}$ are the following square matrices:

$$
\begin{gathered}
\mathbf{U}=\left[\begin{array}{cc}
0 & i \psi^{*} \\
i \psi & 0
\end{array}\right], \quad \mathbf{J}=\left[\begin{array}{cc}
i & 0 \\
0 & -i
\end{array}\right], \\
\mathbf{V}=\left[\begin{array}{cc}
-i|\psi|^{2} & \psi_{t}^{*} \\
-\psi_{t} & i \mid \psi^{2}
\end{array}\right],
\end{gathered}
$$

while $\mathbf{R}$ is a column matrix

$$
\mathbf{R}=\left[\begin{array}{l}
r \\
s
\end{array}\right]
$$

with dependent variables $r=r(x, t)$ and $s=s(x, t)$, and $l$ is a complex eigenvalue. Indeed, it is easy to check that the condition of compatibility

$$
\mathbf{R}_{t x}=\mathbf{R}_{x t}
$$

of the linear equations leads directly to NLSE (1).

To make the calculations more transparent, we can separately write down each component of the vector [Eqs. (2)]:

$$
\begin{gathered}
r_{t}=i \psi^{*} s+i l r, \\
s_{t}=i \psi r-i l s, \\
r_{x}=i l^{2} r+i l \psi^{*} s-\frac{i}{2}|\psi|^{2} r+\frac{1}{2} \psi_{t}^{*} s, \\
s_{x}=-i l^{2} s+i l \psi r-\frac{1}{2} \psi_{t} r+\frac{i}{2}|\psi|^{2} s,
\end{gathered}
$$

Equation (2) and Eq. (6) establish a one-to-one correspondence between the solution of the NLSE, $\psi$, and the solutions of the linear system, $r$ and $s$. The linear system can be solved with ease for the case of trivial solutions of the NLSE, such as the zero solution $\left(\psi_{0}=0\right)$ or the plane-wave solution $\left[\psi_{0}\right.$ $=\exp (i x)]$. In order to deal with more complicated solutions, we can start with one of the above as a "seeding solution" and use it with Darboux transformations to obtain more complicated ones. The zero solution allows us to construct the hierarchy of multisoliton solutions [25], while the planewave solution results in the hierarchy of solutions related to modulation instability [26]. Rational solutions have never been constructed in this way. Instead, they have been obtained as limiting cases of Ma solitons or the so-called Akhmediev breathers. However, for higher-order rational solutions, these limits are highly complicated. Thus, the best way to construct them is to use the Darboux scheme or its equivalent right from the beginning. This will be the aim of the present paper.

\section{HIERARCHY OF RATIONAL SOLUTIONS}

Of course, if we have a core solution of the NLSE, then we can apply scaling and Galilean transforms to obtain "families" of solutions [5]. Hence, in this paper we present only the core solutions since it is simple to apply the above transforms to them to allow "magnification" and nonzero velocities.

Equations (6) have a complex eigenvalue $l$ that defines a parameter of the solution of the NLSE to be derived at the next step of the scheme. Generally, this is any number in the upper half plane of the complex plane. If we restrict ourselves to rational solutions, then the choice of the eigenvalue is very specific. Namely, it has to be $l=i$. We modify the procedure outlined in [26] to find the required solutions of the NLSE. For the origin of the scheme itself, we refer to the book of Matveev and Salle [24]. We use a plane wave as a seeding solution of the NLSE,

$$
\psi_{0}=\exp (i x) .
$$

Our next step is to find two linear functions $r=r(x, t)$ and $s$ $=s(x, t)$ that make system (6) compatible with $\psi=\psi_{0}$. Here, the functions $r, s$ can be multiplied by an arbitrary constant as they are solutions of the linear set of equations.

Generally, solutions of Eq. (6) can be written in exponential functions [11]. Solutions related specifically to rational functions can be obtained as limiting cases of these exponential solutions when the eigenvalue has a limiting value $i$. However, instead of doing this, we introduce an analysis to find the functions and just use the limiting case to verify our results (see Sec. VII).

To start with, we split the functions $\psi(x, t), r_{j}, s_{j}$ into their real and imaginary parts and factor out the exponentials:

$$
\begin{gathered}
\psi(x, t)=e^{i x} \phi(x, t)=\left[\phi_{j r}(x, t)+i \phi_{j i}(x, t)\right] e^{i x}, \\
r_{j}(x, t)=\left[r_{j r}(x, t)+i x r_{j i}(x, t)\right] \exp (-i x / 2), \\
s_{j}(x, t)=\left[s_{j r}(x, t)+i x s_{j i}(x, t)\right] \exp (i x / 2),
\end{gathered}
$$

where index $j$ is related to the order of the solution in the hierarchy, while subscripts $r$ and $i$ mean the real and imaginary parts, respectively. Then the functions $\phi_{j}(x, t), r_{j r}(x, t)$, $r_{j i}(x, t)$ do not contain exponentials. In this paper, $j$ is called "the order of solution" although we stress that it is different from the definition of the order given in [27].

Now we introduce an important simplification obtained from the following mirror symmetries of the functions:

$$
s_{j r}(x, t)=x r_{j i}(x,-t),
$$




$$
x s_{j i}(x, t)=r_{j r}(x,-t) .
$$

We denote $\hat{r}_{j r}(x, t)=r_{j r}(x,-t)$ and $\hat{r}_{j i}(x, t)=r_{j i}(x,-t)$. Once $r_{j}(x, t)$ is fully specified, we have all required functions. As mentioned, any of the functions $r_{j}, s_{j}$ can be multiplied by a constant and it will still give the same solution, $\psi_{j}(x, t)$.

The modified Darboux scheme is

$$
\psi_{0} \rightarrow\left(r_{1}, s_{1}\right) \rightarrow \psi_{1} \rightarrow\left(r_{2}, s_{2}\right) \rightarrow \psi_{2} \rightarrow\left(r_{3}, s_{3}\right) \rightarrow \psi_{3} \cdots .
$$

All the linear functions $r$ and $s$ in this scheme are found by directly solving the linear set of Eqs. (6) with $\psi$ function found at the previous step while the solutions of the NLSE $\psi$ are defined explicitly through the corresponding linear functions.

The first of Eqs. (6) is now

$$
\frac{\partial}{\partial t} r_{j r}(x, t)+r_{j r}(x, t)=x \phi_{j-1, i} \hat{r}_{j i}(x, t)-\phi_{j-1, r} \hat{r}_{j r}(x, t),
$$

while the second one becomes

$$
x \frac{\partial}{\partial t} r_{j i}(x, t)+x r_{j i}(x, t)=x \phi_{j-1, r} \hat{r}_{j i}(x, t)+\phi_{j-1, i} \hat{r}_{j r}(x, t) .
$$

The solution must also satisfy

$$
\begin{aligned}
\frac{\partial}{\partial x} r_{j r}(x, t)= & \frac{x}{2} r_{j i}(x, t)-x \phi_{j-1, r} \hat{r}_{j i}(x, t)-\phi_{j-1, i} \hat{r}_{j r}(x, t) \\
& +\frac{x}{2}\left(\phi_{j-1, r}^{2}+\phi_{j-1, i}^{2}\right) r_{j i}(x, t)+\frac{x}{2} \hat{r}_{j i}(x, t) \frac{\partial}{\partial t} \phi_{j-1, r}(x, t) \\
& +\frac{1}{2} \hat{r}_{j i}(x, t) \frac{\partial}{\partial t} \phi_{j-1, i}(x, t) .
\end{aligned}
$$

The final equation of the set is

$$
\begin{aligned}
x \frac{\partial}{\partial x} r_{j i}(x, t)= & -\frac{1}{2} r_{j r}(x, t)-r_{j i}(x, t)-\phi_{j-1, r} \hat{r}_{j r}(x, t) \\
& +x \phi_{j-1, i} \hat{r}_{j i}(x, t)+\frac{1}{2}\left(\phi_{j-1, r}^{2}+\phi_{j-1, i}^{2}\right) r_{j r}(x, t) \\
& -\frac{x}{2} \hat{r}_{j i}(x, t) \frac{\partial}{\partial t} \phi_{j-1, i}(x, t)+\frac{1}{2} \hat{r}_{j r}(x, t) \frac{\partial}{\partial t} \phi_{j-1, r}(x, t) .
\end{aligned}
$$

Starting with the lowest order $j=1$, we have $\phi_{0}=1$, and the first-order linear differential Eqs. (13) and (14) reduce to

$$
\begin{gathered}
\frac{d}{d t} r_{1 r}+r_{1 r}=-\hat{r}_{1 r}, \\
\frac{d}{d t} r_{1 i}+r_{1 i}=\hat{r}_{1 i} .
\end{gathered}
$$

As we are interested in rational solutions of the lowest order, it is an easy task to find the required functions: $r_{1 r}(x, t)$ $=k_{1}\left(t-\frac{1}{2}\right)$ and $r_{1 i}(x, t)=k_{1}$. Here $k_{1}$ can be any constant, and we choose it to be $\sqrt{2}$ for convenience. Hence

$$
\begin{aligned}
& r_{1}(x, t)=\sqrt{2}\left[t-\frac{1}{2}+i x\right] \exp (-i x / 2), \\
& s_{1}(x, t)=\sqrt{2}\left[x-i\left(t+\frac{1}{2}\right)\right] \exp (i x / 2) .
\end{aligned}
$$

Again, $r_{j r}$ and $r_{j i}$ can always be multiplied by the same constant without affecting the solution. Clearly, writing the solutions of Eq. (6) as rational functions from the beginning is the easiest way to obtain rational solutions of the NLSE.

To obtain the higher-order solutions of the hierarchy, we still use the Darboux expression for $\psi_{j}$ :

$$
\psi_{j}=\psi_{j-1}-\frac{4 i s_{j} r_{j}^{*}}{n_{j}},
$$

where $n_{j}=\left|r_{j}\right|^{2}+\left|s_{j}\right|^{2}$ in the lowest-order case is $n_{1}=1+4 t^{2}$ $+4 x^{2}$.

For any order, the most convenient way to write the solution is the following:

$$
\psi_{j}(x, t)=\left[(-1)^{j}+\frac{G_{j}(x, t)+i x H_{j}(x, t)}{D_{j}(x, t)}\right] \exp [i x],
$$

where $G, H$, and $D$ are polynomials in the two variables $x$ and $t$. The solution remains valid if it is multiplied by $e^{i \theta}$ with $\theta$ real. To be specific, we take $\psi_{j}(0,0)$ to be real and positive. This means that we have $\psi_{j}(0, t) \rightarrow(-1)^{j}$ as $t$ $\rightarrow \pm \infty$. Now the vector $\left(r_{1}, s_{1}\right)$ above allows us to find $\psi_{1}$ directly: we obtain $G_{1}=4, H_{1}=8$, and $D_{1}=n_{1} D_{0}=1+4 t^{2}$ $+4 x^{2}$, where we have set $D_{0}=1$. Thus we get $\psi_{1}$ $=\left[4 \frac{1+2 i x}{1+4 t^{2}+4 x^{2}}-1\right] \exp [i x]$. We can multiply this by -1 , and we then obtain the solution as it is more commonly written as [1]

$$
\psi_{1 a}=\left[1-4 \frac{1+2 i x}{1+4 t^{2}+4 x^{2}}\right] \exp [i x]
$$

For the next step, the standard Darboux scheme gives explicit formulas for $r_{2}$ and $s_{2}$. However, in our case, these expressions fail as all the eigenvalues in the scheme are the same. To apply the scheme, the eigenvalues should be different.

Instead, we solve the linear equations with the $\psi$ function found at the previous step, $\psi=\psi_{1}$, to obtain $r_{2}, s_{2}$. This seems to be complicated as the complexity of these functions increases at each step. However, knowing that the functions are rational means that we can still solve the equations explicitly at higher order if we use a suitable procedure. Calculations can be relatively simple as we expect the denominators of $r_{j}, s_{j}$ to be the same as that of $\psi_{j-1}$ so we use a polynomial (in two variables) divided by this denominator, $D_{j-1}$ as an ansatz. Thus, we can take

$$
r_{2 r}=\frac{b(t)+g(x)+p_{1} t x^{2}}{D_{1}},
$$

where $p_{1}$ is a constant and $b(t)$ and $g(x)$ are low-order polynomials (here both are of order 4), and 


$$
r_{2 i}=\frac{h(t)+p_{2} x^{2}+p_{3} t x^{2}}{D_{1}},
$$

where $p_{2}$ and $p_{3}$ are constants and where $h(t)$ turns out to be of order 3 . We substitute in and equate coefficients. This directly gives the following results for the real functions, $r_{2 r}$ and $r_{2 i}$ :

$$
r_{2 r}=\frac{-\frac{3}{8}+\frac{3}{2} t+2 t^{3}-2 t^{4}+3 x^{2}+2 x^{4}+6 t x^{2}}{D_{1} \sqrt{3}}
$$

and

$$
r_{2 i}=\frac{3+3 t-4 t^{3}+4 x^{2}-4 t x^{2}}{D_{1} \sqrt{3}} .
$$

Applying the mirror symmetry condition, we obtain

$$
\hat{r}_{2 r}=x s_{2 i}==\frac{-\frac{3}{8}-\frac{3}{2} t-2 t^{3}-2 t^{4}+3 x^{2}+2 x^{4}-6 t x^{2}}{D_{1} \sqrt{3}} .
$$

Similarly, we obtain the equation for $s_{2 r}$. In these functions, the constant $\sqrt{3}$ has been chosen for later convenience.

The higher-order solution of the NLSE with $j=2$ then is

$$
\psi_{2}=\psi_{1}-4 i \frac{s_{2} r_{2}^{*}}{n_{2}},
$$

where $n_{2}=\left|r_{2}\right|^{2}+\left|s_{2}\right|^{2}$. As $r$ and $s$ are ratios of polynomials, $n_{2}$ is also a ratio of two functions $D_{j}$. Then the second function $D_{2}=n_{2} D_{1}$ is a single polynomial in two variables. This produces the exact second-order solution:

$$
\psi_{2}=\phi_{2} \exp (i x)=\left[1+\frac{G_{2}+i x H_{2}}{D_{2}}\right] \exp (i x),
$$

where

$$
\begin{aligned}
& G_{2}=\frac{3}{8}-3 t^{2}-2 t^{4}-9 x^{2}-10 x^{4}-12 t^{2} x^{2}, \\
& H_{2}=\frac{15}{4}+6 t^{2}-4 t^{4}-2 x^{2}-4 x^{4}-8 t^{2} x^{2},
\end{aligned}
$$

and

$$
\begin{aligned}
D_{2}= & \frac{1}{8}\left[\frac{3}{4}+9 t^{2}+4 t^{4}+\frac{16}{3} t^{6}+33 x^{2}+36 x^{4}+\frac{16}{3} x^{6}-24 t^{2} x^{2}\right. \\
& \left.+16 t^{4} x^{2}+16 t^{2} x^{4}\right] .
\end{aligned}
$$

This solution has been presented and illustrated previously in [1].

The procedure can be continued to obtain the whole hierarchy of rational solutions $\psi_{j}$. The denominator $D_{j}$ in $\psi_{n}$ appears again in the factors $r_{j+1}, s_{j+1}$ which are used to find $\psi_{j+1}$. We solve the set of equations from the matrix each time, with the previous value of $\psi_{j}$ being used.

\section{THIRD-ORDER SOLUTION}

Applying the above technique, we find the first function $r_{3 r}$ being given by

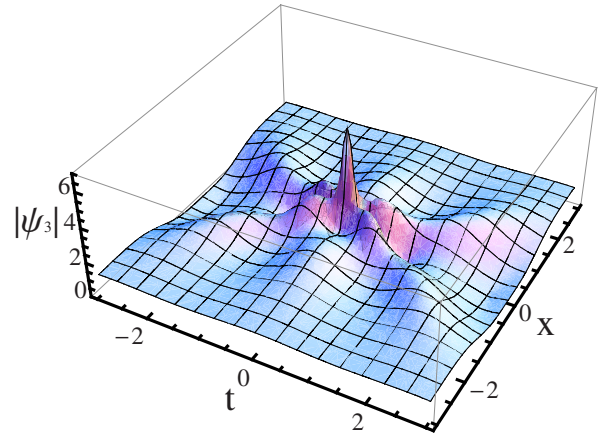

FIG. 1. (Color online) Third-order rational soliton [Eq. (26)]

$$
r_{3 r}=\sqrt{\frac{3}{2}} \frac{f_{0}(t) / 16+x^{2} f_{2}(t)+f_{b}(x, t)}{D_{2}},
$$

where the polynomials $f_{0}(t), f_{2}(t)$, and $f_{b}(x, t)$ are more cumbersome than before and thus are moved to Appendix A. Similarly,

$$
r_{3 i}=\sqrt{\frac{3}{2}} \frac{m_{0}(t)+x^{2} m_{2}(t)+m_{b}(x, t)}{D_{2}},
$$

where the expressions for $m_{0}(t), m_{2}(t)$, and $m_{b}(x, t)$ are given in Appendix B. Now $n_{3}=\left|r_{3}\right|^{2}+\left|s_{3}\right|^{2}$ is a ratio of two functions, while $D_{3}=n_{3} D_{2}$ is a single polynomial.

We find the next-order solution of the NLSE in the form (Fig. 1)

$$
\psi_{3}(x, t)=\left[-1+\frac{G_{3}+i x H_{3}}{D_{3}}\right] \exp (i x) .
$$

Here

$$
\begin{aligned}
& G_{3}(x, t)=\sum_{n=0}^{5} g_{2 n}(t)(2 x)^{2 n}, \\
& H_{3}(x, t)=\sum_{n=0}^{5} h_{2 n}(t)(2 x)^{2 n},
\end{aligned}
$$

and denominator

$$
D_{3}(x, t)=\sum_{n=0}^{6} d_{2 n}(t) x^{2 n},
$$

where polynomials $g_{2 n}(t), h_{2 n}(t)$, and $d_{2 n}(t)$ are given in Appendixes C-E, respectively. Now, it is a straightforward exercise to verify that $\psi_{3}(x, t)$ satisfies the NLSE.

The shape of the central peak of the wave at $x=0$ along the $t$ axis is particularly interesting as it can be compared with the results of numerical simulations with random initial conditions in Sec. VII. Indeed, when $x=0$, the third-order solution is greatly simplified and becomes real:

$$
\phi_{3}(0, t)=\psi_{3}(0, t)=-1+\frac{G_{3}(0, t)}{D_{3}(0, t)}=-1+\frac{g_{0}(t)}{d_{0}(t)},
$$

where polynomials $g_{0}(t)$ and $d_{0}(t)$ are given in Appendixes $\mathrm{C}$ and $\mathrm{E}$ by Eqs. (C2) and (E2), respectively. The maximum 


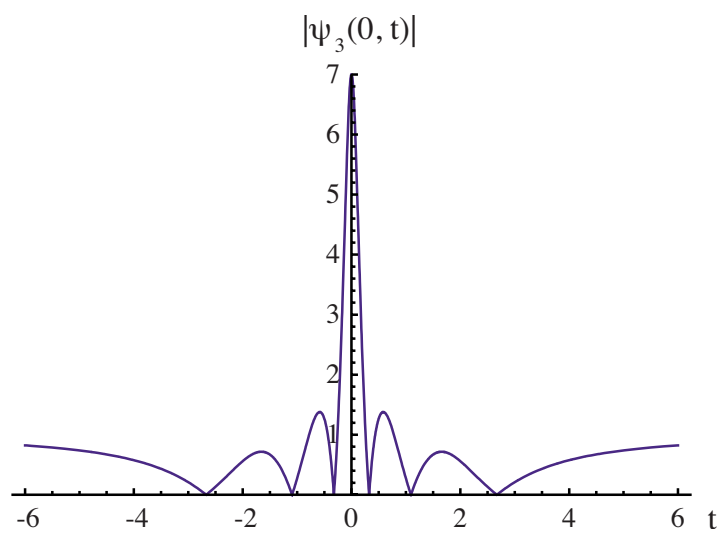

FIG. 2. (Color online) Magnitude of rational solution, $\left|\psi_{3}(0, t)\right|$, on the central line $x=0$ as given by Eq. (30). On this line the field is real and its maximum value is 7 .

value of the main peak of the third-order solution is $\psi_{3}(0,0)=\frac{g_{0}(0)}{d_{0}(0)}-1=7$.

The function $\left|\psi_{3}(0, t)\right|$ is shown in Fig. 2. It has six zeros symmetrically located on the $t$ axis. They occur at approximately $\pm 0.323786, \pm 1.09307, \pm 2.67136$. Thus $\left|\psi_{3}(0, t)\right|$ has five local maxima. The central peak has amplitude of 7 , the two second side peaks have amplitudes of about 1.4, and the two third ones have amplitude of 0.7 .

\section{FOURTH-ORDER SOLUTION}

The complexity of higher-order solutions grows quickly with increasing order. To avoid pages full of equations, for the fourth-order solution we just present it on the middle line $x=0$ where the amplitude profile reaches its maximum. Thus, continuing on, we find that $r_{4 r}(0, t) D_{3}$ is a polynomial of order 16:

$$
r_{r 4}(0, t)=\frac{1}{4 D_{3}} \sum_{n=0}^{16} u_{n}(2 t)^{n},
$$

with the coefficients $u_{n}$ given in Appendix F. The solution itself on the line $x=0$ is purely real:

$$
\psi_{4}(0, t)=1+\frac{G_{4}(0, t)}{D_{4}(0, t)} .
$$

Here

$$
\begin{gathered}
G_{4}(0, t)=8 \sum_{n=0}^{9} q_{n}(2 t)^{2 n}, \\
D_{4}(0, t)=\sum_{n=0}^{10} c_{n}(2 t)^{2 n},
\end{gathered}
$$

and the coefficients $q_{n}$ and $c_{n}$ are given in Appendixes $\mathrm{G}$ and $\mathrm{H}$, respectively. The maximum value is clearly $\psi_{4}(0,0)=1$ $+\frac{G_{4}(0,0)}{D_{4}(0,0)}=1+8=9$. We plot the magnitude of the fourth-order solution $\left|\psi_{4}\right|$ on the central line $x=0$ in Fig. 3 .

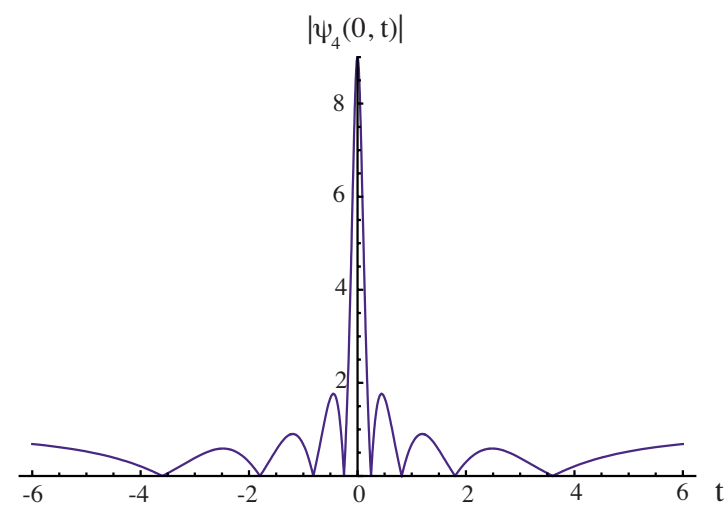

FIG. 3. (Color online) Magnitude of rational solution, $\left|\psi_{4}(0, t)\right|$, on the central line $x=0$, as defined by Eq. (31). On this line, $x=0$, the field is purely real. The maximum value of the amplitude profile is 9 .

\section{GENERAL FEATURES OF THE SOLUTIONS}

We now can make an overview of the main features of the functions we have found. The results are summarized in Table I.

As we progress from $j=1$ to $j=2$ and then $j=3,4$, we find that the highest power appearing in function $r_{j r} D_{j-1}$ is $j^{2}$. The highest power occurring in the function $G_{j}$ and also in $H_{j}$ is $(j-1)(j+2)$. Furthermore, the highest power in $D_{j}$ is $j(j$ $+1)$, while the field $\psi_{j}(0, t)$ for large $t$ approaches $(-1)^{j}$, the central maximum, $\left|\psi_{j}(0,0)\right|$ is equal to $2 j+1$ and the number of zeros is $2 j$. We are always interested in magnitudes so the background level (of deep ocean, etc.) is always 1. These characteristics are summarized in Table I. There is a clear pattern to the number of terms in the main functions. This can be seen in Table II.

\section{A. Integral relations}

We note that [1]

TABLE I. Summary of characteristic parameters of rational solutions presented in this paper. Here $r_{j} D_{j-1}$ : $\exp$ means the highest exponent occurring in the polynomial $r_{j r} D_{j-1}$; for the orders given, it is $j^{2}$. Next, $G, H$ : exp means the highest exponent occurring in the polynomials $G_{j}$ and $H_{j}$; here, it is $(j-1)(j+2)$. Further, $D$ : exp means the highest exponent occurring in $D_{j}$; for the orders given, it is $j(j+1)$. The next column gives the number of zeros in function $\psi_{j}(0, t)$; this is $2 j$ for the cases here. The final column gives the maximum amplitude of function $\psi_{j}(0, t)$; it occurs at the origin and is $\psi_{j}(0,0)=2 j+1$.

\begin{tabular}{cccccc}
\hline \hline & & & \multicolumn{3}{c}{ Number } \\
$j$ & $r_{j} D_{j-1}: \exp$ & $G, H: \exp$ & $D: \exp$ & of zeroes & Max. ampl. \\
\hline 1 & 1 & 0 & 2 & 2 & 3 \\
2 & 4 & 4 & 6 & 4 & 5 \\
3 & 9 & 10 & 12 & 6 & 7 \\
4 & 16 & 18 & 20 & 8 & 9 \\
$j$ & $j^{2}$ & $(j-1)(j+2)$ & $j(j+1)$ & $2 j$ & $2 j+1$ \\
\hline \hline
\end{tabular}


TABLE II. Summary of number of terms appearing in the main functions. For the cases given here, the total number of separate terms in $G_{j}$ (of course, a few of the coefficients equal zero) varies as $\frac{j}{8}(j+1)\left(j^{2}+j+2\right)$. The function $H_{j}$ has the same number. The number of terms in $D_{j}$ follows $\frac{1}{8}\left(j^{2}+j+2\right)\left(j^{2}+j+4\right)$.

\begin{tabular}{ccc}
\hline \hline$j$ & Terms in $G_{j}$ & Terms in $D_{j}$ \\
\hline 1 & 1 & 3 \\
2 & 6 & 10 \\
3 & 21 & 28 \\
\hline \hline
\end{tabular}

$$
\int_{-\infty}^{\infty}\left[\left|\psi_{j}(x, t)\right|^{2}-1\right] d t=0
$$

for all $x$ so that energy is conserved and equals that of the background sea level, i.e., $\left|\psi_{j}(x, t)\right|^{2}$ is equally above and below the mean sea level.

We now present a different interesting relation. Clearly, $\psi_{j}(x, t)-(-1)^{j}$ approaches zero when $x$ and/or $t$ become large. Thus we can define

$$
N_{j}=\int_{-\infty}^{\infty}\left[\psi_{j}(0, t)-(-1)^{j}\right] d t=\int_{-\infty}^{\infty} \frac{G_{j}(0, t)}{D_{j}(0, t)} d t .
$$

Now the integrand for $j=1$ has no zeros, and we easily find that $N_{1}=2 \pi$. In fact, this integrand has a shape resembling the function $4 \operatorname{sech}(2 t)$, which is the fundamental soliton of the NLSE and which also has an integral equal to $2 \pi$.

We now apply complex analysis to determine the $N_{j}$ for $1 \leq j \leq 4$. Now $D_{j}(0, t)$ is of order $j(j+1)$ (see Table I) and has $j(j+1) / 2$ simple zeroes in the upper half of the complex $t$ plane. Each provides a pole of $G_{j}(0, t) / D_{j}(0, t)$, and we can thus express $N_{j}$ as $2 \pi i$ times the sum of the residues at these poles. We find that each residue is $\pm i$, and they can easily be added to obtain $N_{2}=-2 \pi, N_{3}=4 \pi$, and $N_{4}=-4 \pi$. The fact that these integrals, involving ratios of apparently complicated polynomials of order of up to 20 , turn out to be integer multiples of $\pi$ again highlights the point that these solutions are new fundamental objects.

\section{UNCOVERING RATIONAL SOLUTIONS IN CHAOTIC FIELDS}

Now there is a major question: are rational solutions useful in practice or can they only be viewed as an interesting set of mathematical functions? We demonstrate here that physical realizations do occur. Indeed, it turns out that the first-order Peregrine solution is a prototype for the mysterious rogue waves in the ocean [2]. Clearly, higher-order solutions can, very likely, explain rogue waves of even higher amplitudes [1]. Direct confirmation of this conjecture comes from numerical simulations of the NLSE with initial conditions in the form of a plane wave with randomly perturbed amplitude [11]. Specifically, the wave regions of highest amplitude almost coincide in shape with the major peaks of the rational solutions given here. It would be nice to confirm that this indeed happens for even higher-order rational solutions.

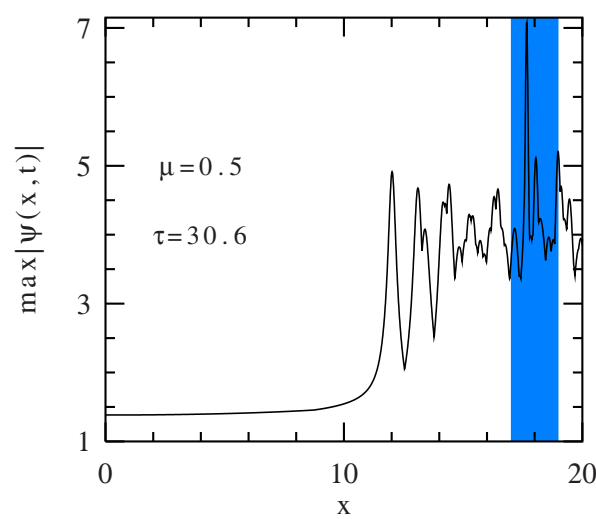

FIG. 4. (Color online) Maximum value of the field amplitude versus $x$ for one of many realizations, showing the appearance of wave sections with an unexpectedly high maximum from a random initial condition. The highest maximum in this simulation is around 7.

In this section we do confirm that this is the case for the third-order rational solution. In principle, the same could be done with the fourth and generally jth-order solutions. However, it is unlikely that the state of ocean surface excitation could be so high.

Thus, we have solved Eq. (1), taking the initial conditions to be a plane wave of amplitude one plus random noise:

$$
\psi(x=0, t)=[1+\mu f(t)],
$$

where $f(t)$ is a normalized complex function, whose real and imaginary parts are independent random functions, uniformly distributed in the interval $[-1,1]$, each with a Gaussian correlation function characterized by its correlation length, $\tau$. As the function $f(t)$ is normalized, the state of excitation of the ocean surface can be described by the coefficients $\mu$ and $\tau$. A number of examples have previously been considered in Ref. [11]. As we are interested in the role of higher-order rational solutions in wave excitations and these occur with very low probability, we needed to perform a large number of numerical simulations for many realizations with different values of $\tau$ and $\mu$ to detect a single case.

A wide numerical grid is used to observe the formation of a large number of interacting waves inside of it. In most of the cases, we used 65536 grid points to cover the temporal interval $[-500,500]$. In order to look for the waves of highest amplitude, we monitored the maximum value of the field amplitude at each value of $x$. As a next step, we made plots of these maxima versus $x$. This two-step procedure allowed us to find the absolute maximum of the wave in two dimensions. Clearly, the absolute maximum depends strongly on the initial state of ocean excitations $\mu$ and $\tau$. For the present study, we adjusted $\mu$ in such a way that the maximum amplitude of 7 could be reached.

Figure 4 shows the results of the simulations described above. Starting with small random perturbations requires a relatively long distance, $x$, which in this case is around 10 , for exponentially growing perturbations to develop. The ocean surface afterwards arrives at an "excited state." Around $x=18$, the absolute maximum of 7 is reached, as can 


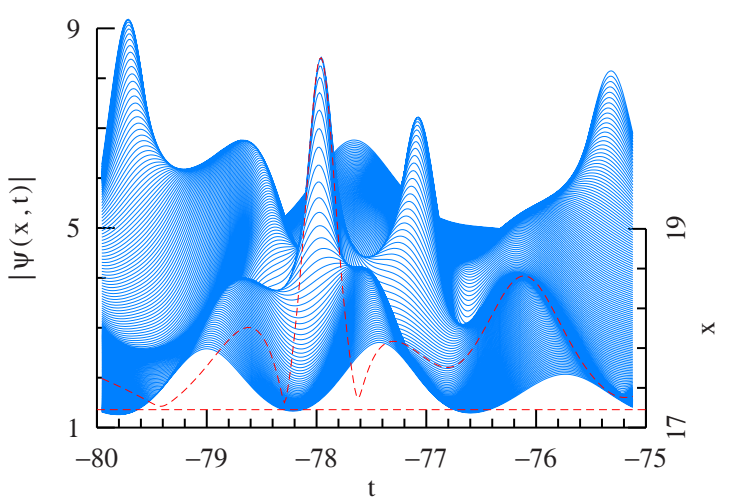

FIG. 5. (Color online) Wave field near the region with the highest maximum, viz. 7 (shaded blue region in Fig. 4). The red dashed curve shows the $t$ dependence at the value of $x$ where the maximum is reached. The horizontal red dashed line indicates, for this profile, the zero amplitude.

be seen from Fig. 4. The remaining part of the curve does not reach such high values and has been removed from this figure.

Part of the curve in Fig. 4 is enclosed in a blue interval. For this interval of $x$ values, the actual wave evolution is shown in Fig. 5. Again, we show here only a small interval of $t$ values that encloses the section of the wave with the highest amplitude. The wave profile around that peak is shown in Fig. 4 by the dashed red curve. Generally speaking, the profile is chaotic. However, the central peak of this chaotic profile has a special shape. In order to show this, we repeat this profile, as a blue dashed line in Fig. 6.

For the sake of comparison, in the same figure, we plot the profile given by the magnitude envelope of the rational solution of the third order, $\left|\phi_{3}(0, t)\right|$, obtained from Eq. (30). The latter is shown as a dotted red line. A direct comparison of these two profiles show that the central peaks and the surrounding two side zeroes perfectly coincide. Of course, we cannot expect the field away from this to follow the exact

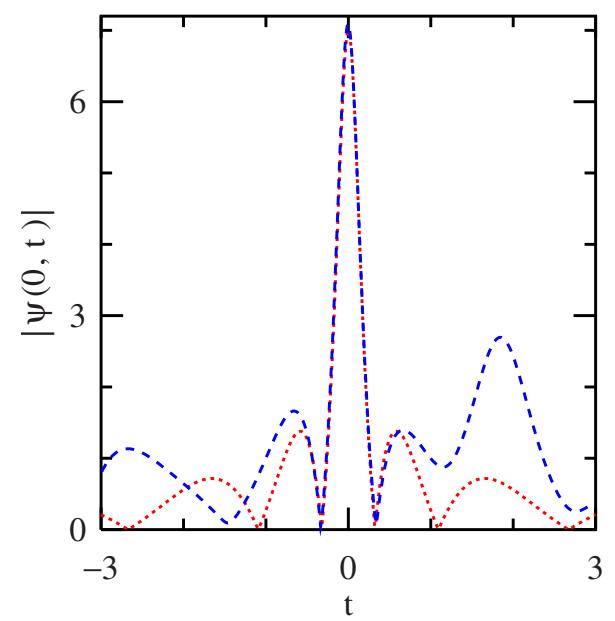

FIG. 6. (Color online) Comparison of the profiles. The dotted red curve represents the magnitude of the exact third-order rational solution, $\left|\phi_{3}(0, t)\right|[$ Eq. (30)]. The dashed blue curve is obtained from numerical simulations with random initial conditions. The central peak and the two main zeroes virtually coincide. solution, as the rest of the wave field is a nonlinear superposition of the third-order solution with chaotic part of the field. However, the profile of the main peak shows clearly that rational solutions play a major role in the formation of rogue waves.

We have no doubt that whenever the highest amplitude of the wave field reaches the values 9, 11, etc., it would be defined by the rational solutions of the corresponding order $(4,5$, etc.). However, we leave detailed studies confirming this claim for the future.

\section{CONCLUSIONS}

In conclusion, we have presented a method for finding rational solutions of the NLSE and given the explicit forms for low-order solutions from the first up until the fourth. While the $j=1$ case [3] and the $j=2$ case $[1,7]$ have been presented before, the $j=3$ and $j=4$ ones are completely new. We have shown that a whole hierarchy of such solutions exists and have provided the method for finding the solution for any $j$ in that hierarchy. We have shown that these solutions may appear as the highest amplitude parts of a chaotic wave field. The possible applications for our results are (1) explanation of rogue waves in the deep ocean and (2) creation of high intensity rogue light wave pulses in optical fibers.

\section{ACKNOWLEDGMENTS}

N.A. and A.A. gratefully acknowledge the support of the Australian Research Council (Discovery Project No. DP0985394). J.M.S.C. acknowledges support from the Spanish Ministerio de Ciencia e Innovación under Contract No. FIS2006-03376.

\section{APPENDIX A: $f$ POLYNOMIALS}

$$
\begin{gathered}
f_{0}(t)=1-3(2 t)-\frac{8}{3}(2 t)^{3}+2(2 t)^{4}-\frac{2}{15}(2 t)^{5} \\
+\frac{8}{45}(2 t)^{6}+\frac{(2 t)^{8}}{45}-\frac{(2 t)^{9}}{3^{3} \cdot 5}, \\
f_{2}(t)=-3-5(2 t)-\frac{2}{3}(2 t)^{3}-\frac{1}{3}(2 t)^{4}+\frac{1}{3 \times 5}(2 t)^{5} \\
+\frac{2}{3^{2} \cdot 5}(2 t)^{6}, \\
f_{b}(x, t)=\left[\begin{array}{l}
\left.10+6(2 t)-\frac{8}{9}(2 t)^{3}+\frac{2}{9}(2 t)^{4}+\frac{2}{3^{2} \cdot 5}(2 t)^{5}\right] x^{4} \\
+
\end{array}\right. \\
+\frac{16 x^{6}}{3^{3} \cdot 5}\left[-93+9(2 t)+2(2 t)^{3}\right]+\frac{16 x^{8}}{3^{2} \cdot 5}(2 t-3) .
\end{gathered}
$$




\section{APPENDIX B: $\boldsymbol{m}$ POLYNOMIALS}

$$
\begin{gathered}
m_{0}(t)=-\frac{9}{8}-2 t+\frac{2}{3}(2 t)^{3}+\frac{(2 t)^{4}}{12}+\frac{(2 t)^{5}}{15}+\frac{(2 t)^{6}}{45}-\frac{(2 t)^{8}}{360}, \\
m_{2}(t)=2\left[-\frac{19}{3}+6(2 t)+\frac{(2 t)^{4}}{3}+\frac{2}{45}(2 t)^{5}-\frac{2}{135}(2 t)^{6}\right], \\
m_{b}(x, t)=4 x^{4}\left[-\frac{7}{5}+\frac{28}{15}(2 t)+\frac{8}{45}(2 t)^{3}-\frac{(2 t)^{4}}{45}\right] \\
+\frac{32 x^{6}}{45}(3+4 t)+\frac{32 x^{8}}{135} .
\end{gathered}
$$

\section{APPENDIX C: $g_{2 n}$ POLYNOMIALS}

We have for $G_{3}(x, t)$

$$
\begin{aligned}
G_{3}= & \sum_{n=0}^{5} g_{2 n}(t)(2 x)^{2 n} \\
= & g_{0}(t)+(2 x)^{2} g_{2}(t)+(2 x)^{4} g_{4}(t)+(2 x)^{6} g_{6}(t)+(2 x)^{8} g_{8}(t) \\
& +(2 x)^{10} g_{10},
\end{aligned}
$$

where

$$
\begin{gathered}
g_{0}(t)=1-(2 t)^{2}-\frac{2}{3}(2 t)^{4}+\frac{14}{45}(2 t)^{6}+\frac{(2 t)^{8}}{45}+\frac{(2 t)^{10}}{675}, \\
g_{2}(t)=-3-20(2 t)^{2}+\frac{2}{3}(2 t)^{4}-\frac{4}{45}(2 t)^{6}+\frac{(2 t)^{8}}{45}, \\
g_{4}(t)=2\left(-\frac{17}{3}+5(2 t)^{2}-\frac{(2 t)^{4}}{3^{2}}+\frac{(2 t)^{6}}{3^{3}}\right), \\
g_{6}(t)=\frac{2}{45}\left(73+14(2 t)^{2}+\frac{7}{3}(2 t)^{4}\right), \\
g_{8}(t)=\frac{1}{15}\left[11+(2 t)^{2}\right], \\
g_{10}=\frac{11}{675} .
\end{gathered}
$$

\section{APPENDIX D: $h_{2 n}(t)$ POLYNOMIALS}

We have for $H_{3}(x, t)$

$$
\begin{aligned}
H_{3}= & \sum_{n=0}^{5} h_{2 n}(t)(2 x)^{2 n} \\
= & h_{0}(t)+(2 x)^{2} h_{2}(t)+(2 x)^{4} h_{4}(t)+(2 x)^{6} h_{6}(t)+(2 x)^{8} h_{8}(t) \\
& +(2 x)^{10} h_{10},
\end{aligned}
$$

where

$$
h_{0}(t)=2\left[7+7(2 t)^{2}-2(2 t)^{4}-\frac{2}{3^{2}}(2 t)^{6}-\frac{(2 t)^{8}}{45}+\frac{(2 t)^{10}}{675}\right],
$$

$$
h_{2}(t)=\frac{2}{3}\left[-11-28(2 t)^{2}-2(2 t)^{4}-\frac{28}{45}(2 t)^{6}+\frac{(2 t)^{8}}{45}\right] \text {, }
$$

$$
\begin{gathered}
h_{4}(t)=\frac{4}{15}\left[-107+19(2 t)^{2}-\frac{7}{3}(2 t)^{4}+\frac{(2 t)^{6}}{3^{2}}\right] \\
h_{6}(t)=\frac{4}{45}\left[-29-2(2 t)^{2}+\frac{(2 t)^{4}}{3}\right], \\
h_{8}(t)=\frac{2}{3^{3}}\left[1+\frac{(2 t)^{2}}{5}\right], \\
h_{10}=\frac{2}{675} .
\end{gathered}
$$

\section{APPENDIX E: $d_{2 n}(t)$ POLYNOMIALS}

We have for $D_{3}(x, t)$

$$
\begin{aligned}
D_{3}= & \sum_{n=0}^{6} d_{2 n} x^{2 n} \\
= & d_{0}(t)+x^{2} d_{2}(t)+x^{4} d_{4}(t)+x^{6} d_{6}(t)+x^{8} d_{8}(t)+x^{10} d_{10}(t) \\
& +x^{12} d_{12},
\end{aligned}
$$

where

$$
\begin{aligned}
d_{0}(t)= & \frac{1}{2^{3}}\left[1+6(2 t)^{2}+\frac{5}{3}(2 t)^{4}+\frac{52}{45}(2 t)^{6}+\frac{(2 t)^{8}}{15}+\frac{2}{675}(2 t)^{10}\right. \\
& \left.+\frac{(2 t)^{12}}{2025}\right], \\
d_{2}(t) & =23-9(2 t)^{2}+\frac{10}{3}(2 t)^{4}+\frac{2}{15}(2 t)^{6}-\frac{(2 t)^{8}}{45}+\frac{(2 t)^{10}}{675}
\end{aligned}
$$

$$
d_{4}(t)=2\left[71+\frac{116}{3}(2 t)^{2}-\frac{2}{3}(2 t)^{4}-\frac{4}{45}(2 t)^{6}+\frac{(2 t)^{8}}{135}\right],
$$

$$
\begin{gathered}
d_{6}(t)=\frac{32}{3}\left[\frac{17}{3}+5(2 t)^{2}+\frac{(2 t)^{4}}{45}+\frac{(2 t)^{6}}{135}\right], \\
d_{8}(t)=\frac{32}{15}\left[\frac{83}{3}+2(2 t)^{2}+\frac{(2 t)^{4}}{3^{2}}\right], \\
d_{10}(t)=\frac{2^{8}}{225}\left[7+\frac{(2 t)^{2}}{3}\right],
\end{gathered}
$$




$$
d_{12}=\frac{2^{9}}{2025} .
$$

APPENDIX F: $u_{2 n}(t)$ COEFFICIENTS

$$
\begin{gathered}
u_{0}=1, \quad u_{1}=-4, \quad u_{2}=0, \\
u_{3}=-\frac{20}{3}, \quad u_{4}=\frac{20}{3}, \quad u_{5}=-\frac{4}{5}, \\
u_{6}=\frac{64}{45}, \quad u_{7}=-\frac{4}{315}, \quad u_{8}=\frac{38}{105}, \\
u_{9}=-\frac{4}{27}, \quad u_{10}=\frac{64}{4725}, \quad u_{11}=-\frac{4}{525}, \\
u_{12}=4 / 14175, \quad u_{13}=-u_{12}, \quad u_{14}=0, \\
u_{15}=-4 / 212625, \quad u_{16}=1 / 212625 .
\end{gathered}
$$

\section{APPENDIX G: $q_{2 n}(t)$ COEFFICIENTS}

$$
\begin{gathered}
q_{0}=1, \quad q_{1}=-5, \quad q_{2}=-4, \\
q_{3}=\frac{4}{15}, \quad q_{4}=\frac{34}{315}, \quad q_{5}=\frac{38}{1575}, \\
q_{6}=-\frac{4}{945}, \quad q_{7}=-4 / 19845, \\
q_{8}=-1 / 165375, \quad q_{9}=-1 / 4465125 . \\
\text { APPENDIX H: } c_{2 n}(t) \text { COEFFICIENTS } \\
c_{0}=1, \quad c_{1}=10, \quad c_{2}=5, \\
c_{3}=\frac{88}{15}, \quad c_{4}=\frac{82}{105}, \quad c_{5}=\frac{116}{1575}, \\
c_{6}=\frac{2}{105}, \quad c_{7}=8 / 11025, \quad c_{8}=1 / 55125, \\
c_{9}=2 / 4465125, \quad c_{10}=1 / 22325625 .
\end{gathered}
$$

[1] N. Akhmediev, A. Ankiewicz, and M. Taki, Phys. Lett. A 373, 675 (2009).

[2] V. I. Shrira and V. V. Georgaev, What makes the Peregrine soliton so special as a prototype of freak waves? Submitted (private communication).

[3] D. H. Peregrine, J. Aust. Math. Soc. Ser. B, Appl. Math. 25, 16 (1983).

[4] A. Ankiewicz, (URL of website, 2009): http:// demonstrations.wolfram.com/RogueOceanWaves/

[5] N. Akhmediev and A. Ankiewicz, Solitons, Nonlinear Pulses, and Beams (Chapman and Hall, London, 1997).

[6] Ya.-C. Ma, Stud. Appl. Math. 60, 43 (1979).

[7] N. Akhmediev and V. I. Korneev, Theor. Math. Phys. 69, 1089 (1986) (translation from Russian).

[8] K. B. Dysthe and K. Trulsen, Phys. Scr. T82, 48 (1999).

[9] I. Ten and H. Tomita, Reports of RIAM Symposium No. 17SP1-2, Proceedings of a Symposium Held at Chikushi Campus, Kyushu University, Kasuga, Fukuoka, Japan, 2006 (unpublished), article 04.

[10] V. V. Voronovich, V. I. Shrira, and G. Thomas, J. Fluid Mech. 604, 263-296 (2008).

[11] N. Akhmediev, J. M. Soto-Crespo, and A. Ankiewicz, Phys. Lett. A 373, 2137 (2009).

[12] T. B. Benjamin and J. E. Feir, J. Fluid Mech. 27, 417 (1967).

[13] V. I. Bespalov and V. I. Talanov, Zh. Eksp. Teor. Fiz. Pis'ma Red. 3, 471 (1966) [JETP Lett. 3, 307 (1966)].
[14] P. Müller, Ch. Garrett, and A. Osborne, Oceanogr. (Wash. DC) 18, 66 (2005).

[15] C. Kharif and E. Pelinovsky, Eur. J. Mech. B/Fluids 22, 603 (2003).

[16] A. A. Kurkin and E. N. Pelinovsky, Killer-waves: Facts, Theory, and Modeling (Book in Russian) (Nizhny Novgorod, Russia, 2004).

[17] D. R. Solli, C. Ropers, P. Koonath, and B. Jalali, Nature (London) 450, 1054 (2007).

[18] D-Il. Yeom and B. Eggleton, Nature (London) 450, 953 (2007).

[19] V. E. Zakharov and A. B. Shabat, Sov. Phys. JETP 34, 62 (1972).

[20] A. R. Osborne, Mar. Struct. 14, 275 (2001).

[21] A. Hasegawa and F. Tappert, Appl. Phys. Lett. 23, 142 (1973).

[22] A. N. W. Hone, J. Phys. A 30, 7473 (1997).

[23] P. A. Clarkson, Eur. J. Appl. Math. 17, 293 (2006).

[24] V. B. Matveev and M. A. Salle, Darboux Transformations and Solitons (Springer, Berlin, 1991).

[25] N. Akhmediev and N. V. Mitskevich, IEEE J. Quantum Electron. 27, 849 (1991).

[26] N. Akhmediev, V. I. Korneev, and N. V. Mitskevich, Zh. Eksp. Teor. Fiz. 94, 159 (1988) [JETP Lett. 67, 89 (1988)].

[27] N. Akhmediev, V. M. Eleonskii, and N. E. Kulagin, Theor. Math. Phys. (USSR) 72, 809 (1987) [Teor. Mat. Fiz., 72, 183 (1987)]. 